\title{
Comportamentos Pró-Sociais de Adolescentes em Acolhimento Institucional ${ }^{1}$

\author{
Amanda Oliveira Fernandes ${ }^{2}$ \\ Nancy Ramacciotti de Oliveira Monteiro \\ Universidade Federal de São Paulo
}

\begin{abstract}
RESUMO - Realizou-se um levantamento de indicadores de comportamentos pró-sociais em adolescentes acolhidos. A pesquisa foi realizada com 61 adolescentes (11 a 18 anos; 34 meninas e 27 meninos), que viviam em instituições de acolhimento e foram avaliados por meio da Escala de Medida de Pró-Socialidade (EMPA). A análise dos resultados foi feita por subgrupos: idade (11-14 anos/15-18 anos), sexo e tempo de acolhimento (até dois anos e mais de dois anos). Resultados indicaram tendência a comportamentos pró-sociais de cuidado, principalmente, nos adolescentes com menos tempo de institucionalização. Meninas referiram mais comportamentos pró-sociais do que os meninos. Os participantes indicaram tendência à dificuldade de empatia e de partilha de objetos pessoais e de valor.
\end{abstract}

Palavras-chave: adolescência, acolhimento, comportamento pró-social

\section{Prosocial Behavior among Adolescents in Institutional Sheltering}

\begin{abstract}
It were investigated indicators of prosocial behavior in sheltered adolescents. Participated in the study 61 adolescents (ages 11-18; 34 girls and 27 boys), who lived in shelters and were evaluated by the Measurement Scale ProSociality (EMPA). The analysis of the results considered subgroups of age (11-14/15-18) and sex, as also how long they lived in a shelter (until two years and more than two years). Findings indicated a tendency to prosocial behaviors of care, shown mainly in adolescents who lived a shorter period in shelters. Girls reported more prosocial behavior than boys. Participants indicated tendency to difficulty of empathy and sharing valuable and personal objects.
\end{abstract}

Keywords: adolescence, shelter, prosocial behavior

O período da vida definido como adolescência é considerado como uma importante etapa do desenvolvimento humano, com grandes transformações e reorganizações que atingem múltiplas dimensões, sejam biológicas, afetivoemocionais, comportamentais, cognitivas, morais ou sóciopsico-emocionais (Senna \& Dessen, 2012). Inserida entre a infância e a idade adulta, a adolescência compreende o periodo entre os 10 e 19 anos de idade, segundo parâmetros da Organizacão Mundial de Saúde (Organizacion Mundial de la Salud [OMS], 1999). Caracteriza-se pelo estabelecimento de novos hábitos de conduta e modelos de socialização, bem como pela oportunidade de aquisição de novas habilidades e recursos para lidar com as transições que lhe são próprias. Embora tradicionalmente a adolescência tenha sido tratada pela Psicologia como uma fase caracteristicamente marcada por crises e conflitos, novos estudos têm contestado essa abordagem, apresentando essa etapa da vida com grande potencial para o desenvolvimento positivo (González, Cuéllar, Miguel, \& Desfilis, 2009).

Adolescentes expostos a vivências de risco e/ou violação de direitos relacionados a seus ambientes familiares podem

1 Projeto subsidiado pela Fundação de Amparo à Pesquisa do Estado de São Paulo (FAPESP): processo 2011/04610-2.

2 Endereço para correspondência: Rua Silva Jardim, 136, Vila Mathias, Santos, SP, Brasil. CEP. 11.015-020 E-mail: amanda.psi16@gmail.com ser afastados do convivio familiar, passando a viver em instutições de acolhimento. Conforme o Estatuto da Criança e do Adolescente (ECA; Decreto Lei $n^{\circ}$ 8.069/1990), as instituições de acolhimento existem para oferecer proteção em caráter provisório e excepcional a crianças e adolescentes que tiveram seus direitos violados ou ameaçados e cuja convivência com a família de origem seja considerada prejudicial ao seu desenvolvimento. Segundo a Lei de Adoção (Decreto Lei $n^{\circ} 12.010 / 2009$ ), a permanência da criança ou do adolescente em programa de acolhimento institucional não deve se prolongar por mais de 2 anos. Porém, em alguns casos, a institucionalização se mantém como caminho utilizado indiscriminadamente e, muitas vezes, considerado o único possível para a proteção da infância e da adolescência (Ministério do Desenvolvimento Social e Combate à Fome, 2006).

Dados de 2009 apontavam que o número estimado de crianças e adolescentes moradores de instituições de acolhimento pelo mundo era de mais de oito milhões (Save the Children, 2009). No Brasil, dados de 2011 mostravam que 37 mil crianças e adolescentes estariam vivendo em 2624 serviços de acolhimento institucional, segundo indicadores do Levantamento Nacional das Crianças e Adolescentes em Serviços de Acolhimento (LNCA; Assis \& Farias, 2013).

Para Dell'Aglio e Siqueira (2010), embora o acolhimento seja uma medida de proteção, a institucionalização pode ser vivenciada como um risco para o desenvolvimento. Mesmo sendo temporária, ela tem sido associada, durante a infância 
e a adolescência, a rompimentos de vínculos e a situações de violência. Fernandes, Oliveira-Monteiro, Spadari-Bratfisch, Nascimento e Montesano (2015) referiram que adolescentes com vivência em acolhimento institucional apresentavam situações de estresse em diversos âmbitos da vida, como na escola e em questões de ordem financeira ou jurídicoinstitucional, além de na família. Wathier e Dell'Aglio (2007) colocam que a vida de crianças e adolescentes acolhidos é marcada por muitos eventos adversos. Além disso, aspectos como a alta rotatividade de funcionários nessas instituições e a falta de atividades planejadas ou de apoio afetivo podem trazer prejuízos aos jovens que se desenvolvem em instituições.

Não obstante o distanciamento familiar e a vivência institucional possam gerar prejuízos no desenvolvimento, alguns autores destacam os aspectos positivos e de proteção dos contextos de acolhimento. Os adolescentes acolhidos podem ter uma percepção positiva da instituição e de seus funcionários, pelo atendimento de suas necessidades básicas, como de alimentação e de vestuário (Chapman, Wall, \& Barth, 2004; Siqueira \& Dell'Aglio, 2007), além de oportunidades de lazer, relações de amizade e configurações de vínculos de confiança (Carlos, Ferriani, Silva, Roque, \& Vendruscolo, 2013; Siqueira, Zoltowski, Giordani, Otero, \& Dell'Aglio, 2010). Para crianças e adolescentes institucionalizados, as pessoas com quem passam a conviver e interagir representam suas redes de apoio social e afetivo, fazendo do serviço de acolhimento um importante ambiente no seu desenvolvimento (Yunes, Miranda, \& Cuello, 2004). A institucionalização, enquanto contexto de desenvolvimento, oferece recursos para esses adolescentes que lhes permitem a construção de novas respostas socialmente válidas para lidarem com as adversidades, além do estabelecimento de regras e limites internos e externos facilitados pela aproximação de referenciais positivos (Carlos et al., 2013; Santos \& Bastos, 2002). Fernandes e Oliveira-Monteiro (2016) apontam que a vida em serviços institucionais pode associar-se tanto a fatores positivos como a fatores de risco para o desenvolvimento de crianças e adolescentes, dependendo das particularidades e vulnerabilidades desses ambientes de interação.

Em estudos sobre a adolescência, Steinberg e Lerner (2004) trazem perspectivas de interesse à investigação de aspectos do desenvolvimento positivo do indivíduo. Contribuições da Psicologia Positiva indicam que há fatores protetivos em diferentes níveis ambientais que podem modificar respostas a fatores de risco e a eventos estressores, como as estratégias de coping, a resiliência (Dell' Aglio, Koller, \& Yunes, 2006) e os comportamentos pró-sociais (Sapienza, 2006).

A pró-socialidade é configurada como um conjunto de comportamentos que, sem buscar recompensas externas ou materiais, favorecem outras pessoas ou grupos e aumentam a probabilidade de gerar reciprocidade positiva e solidária nas relações interpessoais ou sociais (Roche, 2007). Comportamentos pró-sociais podem ser motivados por uma série de fatores, incluindo interesses egoístas (desejo de reciprocidade ou aprovação social), preocupações práticas (como o desperdício de bens), outros tipos de questões (como simpatia) ou valores morais (desejo de manter valores morais internos), além de abarcarem uma gama de comportamentos específicos que podem ir da ajuda física ao altruismo (Auné, Blum, Abal, Lozzia, \& Attorresi, 2014; Eisenberg, Eggum, \& Di Giunta, 2010). Segundo Roche (2010), o aumento de ações pró-sociais produz diminuição de comportamentos violentos, auxiliando na consolidação de um tecido social baseado na cultura da empatia, da generosidade, da gratuidade e da solidariedade. Comportamentos como partilha, cuidado, empatia e ajuda são indicados como comportamentos prósociais e têm sido utilizados como itens na mensuração da pró-socialidade (Caprara, Steca, Zelli, \& Capanna, 2005).

Complexos processos do desenvolvimento psicológico e geral estão envolvidos na gênese dos comportamentos prósociais, relacionando-se ao raciocínio moral, às capacidades de aprendizagem, à autoregulação, às influencias familiares e sociais (Auné et al., 2014). Estudo de Vecchione e Picconi (2010) mostrou que respostas pró-sociais tornam-se relativamente estáveis no período do final da infância e de início da adolescência. Assim, modelos de desenvolvimento da pró-socialidade poderiam se apresentar como uma potencial alternativa para intervenção e prevenção junto a crianças e jovens em situação de vulnerabilidade e a mobilização de resiliência, com condições de atingir diversos contextos de interação (Aznar-Farias \& Oliveira-Monteiro, 2006).

Frente às considerações apresentadas, o presente trabalho ${ }^{1}$ propôs realizar um levantamento de indicadores de comportamentos pró-sociais de adolescentes acolhidos, considerando sexo, tempo de acolhimento (até dois anos e mais de dois anos) e faixas etárias (11-14 anos e 15-18 anos).

\section{Método}

\section{Sujeitos}

Foram investigados 61 adolescentes de ambos os sexos (34 do sexo feminino e 27 do sexo masculino), idades de 11 a 18 anos incompletos, que eram moradores de serviços de acolhimento institucional. Os adolescentes investigados apresentavam tempo de acolhimento com variação de uma semana a mais de oito anos.

A amostra foi constituída por conveniência, considerando concordância institucional para a realização da pesquisa, aceite/disponibilidade dos adolescentes para a participação e o tempo dedicado ao levantamento dos dados no campo (seis meses). Participaram do estudo todos os adolescentes que aceitaram o convite dos pesquisadores, moradores de 10 serviços de acolhimento institucional (governamentais e não-governamentais) da Baixada Santista (SP).

\section{Instrumentos}

Para o levantamento de indicadores de comportamentos pró-sociais, foi utilizada a Escala de Medida de PróSocialidade (EMPA). Esse instrumento consiste em uma escala de autoavaliação de 16 itens com afirmações sobre comportamentos pró-sociais (partilha, ajuda, cuidado e 
empatia). É explicitado que não existem respostas certas ou erradas, sendo a melhor resposta a imediata (espontânea). Cada item apresenta uma escala do tipo Likert de cinco pontos: nunca/quase nunca verdadeiro (1); ocasionalmente verdadeiro (2); algumas vezes verdadeiro (3); frequentemente verdadeiro (4); sempre ou quase sempre verdadeiro (5). O tempo previsto para responder à EMPA é de 15 minutos. Caprara et al. (2005) propõem o agrupamento das 16 questões em quatro grupos referentes a ações pró-sociais: (a) partilha, (b) ajuda, (c) cuidado e (d) empatia. O instrumento foi usado por Roche e sua equipe do Laboratório de Investigação Pró-social Aplicada (LIPA) da Universidade Autónoma de Barcelona (Espanha), no início e na finalização de um programa de intervenção com adolescentes (Roche \& Selva, 2010). O instrumento foi validado semanticamente para o português a partir do trabalho de tradução do instrumento em língua italiana, para o inglês, do inglês para o espanhol e então do espanhol para o português, junto à equipe do LIPA.

\section{Procedimentos}

O estudo foi aprovado pelo Comitê de Ética em Pesquisa da Universidade Federal de São Paulo (CEP n ${ }^{\circ}$ 0313/11). Houve o contato para exposição da proposta da pesquisa e de busca de aceite com responsáveis pelos serviços de acolhimento. A aplicação do instrumento ocorreu mediante a apresentação da proposta da pesquisa, explicação e assinatura do Termo de Consentimento Livre e Esclarecido (TCLE) pelos responsáveis legais (diretores das instituições) e Termos de Assentimento (TA) para os adolescentes, em locais, dias e horários combinados com as equipes dos serviços de acolhimento e com os adolescentes. A duração da aplicação do instrumento foi de, aproximadamente, 15 minutos e realizada individualmente. Após a entrega do instrumento respondido pelos adolescentes, a aplicadora, primeira autora deste artigo, verificava se todos os itens estavam completos nas respostas, havendo orientações em casos necessários.

$\mathrm{O}$ procedimento de tratamento de dados da EMPA considerou um agrupamento das cinco escolhas/graus da escala Likert para três níveis de pró-socialidade, da seguinte forma: menor pró-socialidade para as escolhas graus 1 ou 2 (nuncalquase nunca verdadeiro e ocasionalmente verdadeiro), indiferente para escolhas grau 3 (algumas vezes verdadeiro) e maior pró-socialidade para escolhas graus 4 ou 5 (frequentemente verdadeiro e sempre ou quase sempre verdadeiro). Os itens das 16 questões do instrumento foram agrupados para quatro grupos, conforme proposta dos autores do instrumento: partilha, ajuda, cuidado e empatia. A análise dos resultados da EMPA considerou o cálculo de frequências de respostas para cada nível de pró-socialidade para uma análise descritiva, além de inferencial, através da análise de variância com três fatores fixos. Para estudo da associação entre as respostas dadas em cada questão da EMPA e os grupos de jovens, utilizou-se o teste exato de Fisher. Os resultados foram analisados por subgrupos referentes: (a) à faixa etária (11 a 14 anos/15 a 18 anos), (b) ao sexo e (c) ao tempo de acolhimento (até dois anos/mais de dois anos).

\section{Resultados}

Os resultados da EMPA indicaram frequências altas para maiores índices de pró-socialidade em quase todos os grupos avaliados, ou seja, Partilha, Ajuda e Cuidado. A questão 11 (emprestar dinheiro ou coisas) do grupo Partilha recebeu mais respostas dentro do estudo para o grau menor de prósocialidade (52,5\%). Dentro do grupo Ajuda, referências de maior pró-socialidade apareceram na questão 3 (tentar ajudar as pessoas; $65,6 \%$ ), sendo as maiores do estudo. No grupo Cuidado, a questão 15 (dedicar tempo aos amigos solitários) obteve maior índice de pró-socialidade $(63,9 \%)$, e a questão 4 (oferta para atividades voluntárias) apresentou mais respostas para menor pró-socialidade $(39,3 \%)$.

O grupo Empatia obteve frequências mais altas para menor pró-socialidade em dois de seus quatro itens. A questão 5 (entrar em sintonia com o estado de ânimo de quem sofre) obteve mais referências para o grau menor de pró-socialidade (44,3\%), e a questão 12 (colocar-se no lugar dos necessitados) obteve maior frequência de respostas para o grau maior de pró-socialidade $(47,5 \%)$.

A Tabela 1 apresenta a frequência das respostas dadas em cada questão da EMPA, na amostra, nos quatro grupos de pró-socialidade analisados no instrumento.

A Tabela 2 apresenta as medidas descritivas da EMPA, segundo o sexo. O escore médio das meninas foi de 56,9 e o dos meninos, de 53,2. No total, os adolescentes do estudo apresentaram escore médio de 55,3 para a EMPA.

Quando comparados por faixa etária, no total, os adolescentes de 11 a 14 anos apresentaram média de 53,2 e os de 15 a 18 anos, média de 57,1. As meninas de ambas as faixas etárias obtiveram médias parecidas. As meninas de 11 a 14 anos apresentaram média de 57,7, enquanto que os meninos da mesma faixa etária obtiveram média de 47,2. Os meninos mais velhos (15 a 18 anos) pontuaram a maior média, com 58,1 .

Referente ao tempo de acolhimento, os adolescentes com até dois anos de acolhimento apresentaram média de 55,8 e os com mais de dois anos de acolhimento, média de 54,8. As meninas de ambos os tempos de acolhimento obtiveram médias parecidas. As meninas com mais de dois anos de acolhimento apresentaram média de 57,6, enquanto que os meninos do mesmo grupo obtiveram média de 51,3.

Foi encontrada associação entre o grupo Cuidado e a variável tempo de acolhimento. O escore médio desse grupo, dos adolescentes que tinham até dois anos de acolhimento foi maior do que o daqueles que tinham mais de dois anos de acolhimento.

\section{Discussão}

Considerando a fase da adolescência, interações da vivência em contexto institucional podem ser fatores importantes frente ao desenvolvimento de problemas e recursos positivos de jovens institucionalizados. Resultados encontrados neste estudo apontaram referências de indicadores de comportamentos pró-sociais nos adolescentes 
Tabela 1. Frequência das respostas dadas a cada questão da EMPA na amostra, nos quatro grupos

\begin{tabular}{|c|c|c|c|c|}
\hline & & $\begin{array}{l}\text { Menor pró- } \\
\text { socialidade }\end{array}$ & Indiferente & $\begin{array}{l}\text { Maior pró- } \\
\text { socialidade }\end{array}$ \\
\hline \multirow{4}{*}{ Partilha } & Dividir coisas (Q2) & 26,2 & 21,3 & 52,5 \\
\hline & Disponibilizar conhecimentos e habilidades (Q9) & 24,6 & 18,0 & 57,4 \\
\hline & Emprestar dinheiro ou coisas (Q11) & 52,5 & 24,6 & 23,0 \\
\hline & Dividir boas oportunidades (Q14) & 18,0 & 18,0 & 63,9 \\
\hline \multirow{4}{*}{ Ajuda } & Ajudar amigos/colegas nas atividades (Q1) & 13,1 & 24,6 & 62,3 \\
\hline & Tentar ajudar as pessoas (Q3) & 11,5 & 23,0 & 65,6 \\
\hline & Ajudar imediatamente quem precisa (Q6) & 29,5 & 18,0 & 52,5 \\
\hline & Ajudar pessoas a não se meterem em problemas (Q7) & 18,0 & 18,0 & 63,9 \\
\hline \multirow{4}{*}{ Cuidado } & Oferta para atividades voluntárias (Q4) & 39,3 & 16,4 & 44,3 \\
\hline & Consolar quem está triste (Q10) & 19,7 & 21,3 & 59,0 \\
\hline & Tentar estar próximo e cuidar de quem passa necessidade (Q13) & 24,6 & 26,2 & 49,2 \\
\hline & Dedicar tempo aos amigos solitários (Q15) & 24,6 & 11,5 & 63,9 \\
\hline \multirow{4}{*}{ Empatia } & Entrar em sintonia com o estado de ânimo de quem sofre (Q5) & 44,3 & 16,4 & 39,3 \\
\hline & Sentir intensamente os que os outros sentem (Q8) & 41,0 & 19,7 & 39,3 \\
\hline & Colocar-se no lugar dos necessitados (Q12) & 39,3 & 13,1 & 47,5 \\
\hline & & 32,8 & 26,2 & 41,0 \\
\hline
\end{tabular}

Tabela 2. Medidas descritivas da EMPA, segundo sexo

\begin{tabular}{lcc}
\multicolumn{1}{c}{ Sexo } & Média & Desvio-padrão \\
\hline Feminino & 56,9 & 12,7 \\
Masculino & 53,2 & 11,6 \\
Total & 55,3 & 12,3 \\
\hline
\end{tabular}

investigados em todos os grupos estudados, com algumas nuances de diversidade.

As meninas apresentaram tendência de maior prósocialidade do que os meninos. Esse resultado vai ao encontro de estudos sobre o desenvolvimento do comportamento pró-social em adolescentes, os quais mostraram também que essa diferença se mantém relativamente estável nos anos posteriores (Mestre, Tur, Samper, Nácher, \& Cortés, 2007; Reppold, Gurgel, \& Hutz, 2016; Vecchione \& Picconi, 2010). No que se refere a comportamentos de ajuda, cooperação e envolvimento emocional, as meninas tendem a demonstrar mais ações pró-sociais do que os meninos, envolvendo-se com aspectos emocionais do próximo (Koller \& Bernandes, 1997). Poletto, Koller e Dell'Aglio (2009) apontaram que as meninas são mais propensas a investirem nos relacionamentos interpessoais a longo prazo e a se envolverem com as dificuldades dos outros. Já os meninos teriam tendência a ajudar o próximo em ações mais urgentes e com necessidades claras (Auné et al., 2014). Questões fisiológicas, também, estariam relacionadas a essas diferenças, conforme Cáceda et al. (2014), os hormônios sexuais e as estruturas cerebrais especificas influenciariam comportamentos diferentes de homens e mulheres em relação à pró-socialidade.

Em relação à faixa etária, o desenvolvimento de comportamentos pró-sociais é indicado como sinal de maturidade, havendo correlações positivas entre idade e repertórios pró-sociais (Auné et al., 2014). Neste estudo, os adolescentes de 15 a 18 anos apresentaram tendência a maior pró-socialidade que os mais novos. Segundo Vecchione e Picconi (2010), ao se observar a estabilidade de um comportamento por um longo período de tempo, é importante considerar a relação dos comportamentos nos diferentes momentos do desenvolvimento e suas associações com determinados fatores de risco ou sintomas. O desenvolvimento dos comportamentos pró-sociais ocorre, principalmente, durante a infância, quando repertórios sociais são aprendidos, e é influenciado pelo grupo cultural ao qual a criança pertence, pelas experiências de socialização, pelos processos cognitivos e pelas variáveis pessoais que marcam a vida do indivíduo (Eisenberg \& Musen, 1989). Nesse sentido, cabe ressaltar que crianças e adolescentes moradores de serviços de acolhimento possuem peculiaridades no seu desenvolvimento, consequência de vivências de abandono, maus tratos, violência, falta de vínculos positivos e condições de vulnerabilidade social.

Os adolescentes investigados apresentaram referências de maior pró-socialidade para as questões dos grupos Ajuda, Partilha e Cuidado. Somente a questão referente a emprestar dinheiro ou coisas do grupo Partilha apresentou referências de menor pró-socialidade, indicando dificuldades em empréstimo de coisas materiais, possivelmente, pela própria condição de pobreza e carência de objetos de valor dos adolescentes acolhidos. Dentro do grupo Ajuda, referências de maior pró-socialidade apresentaram-se na questão tentar ajudar as pessoas, com as maiores médias do estudo. Conforme Cavalcante, Costa e Magalhães (2012), a vivência em instituições coletivas e de acolhimento aproxima crianças 
e adultos, o que pode favorecer comportamentos de afeto e ajuda. A investigação dessas autoras sobre comportamento de cuidado em instituições de acolhimento mostrou que a Ajuda foi o comportamento mais referido como realizado pelos acolhidos, associado à ajuda aos pares. No grupo Empatia, colocar-se no lugar dos necessitados foi o comportamento com maiores referências de pró-socialidade, mostrando a presença de um sentimento coletivo de identificação com aqueles que passam por dificuldades.

Dentro do grupo Cuidado, a questão referente a dedicar tempo aos amigos solitários obteve referências de maior pró-socialidade. Nos comportamentos pró-sociais de cuidado e ajuda, crianças se sentem mais motivadas em interações com pessoas necessitadas que sejam conhecidas, amigas ou com características semelhantes (Cavalcante \& Costa, 2011). Neste estudo, no grupo Cuidado, os adolescentes que tinham até dois anos de acolhimento referiram mais pró-socialidade do que aqueles que tinham mais de dois anos de acolhimento. Esse dado pode ser refletido a partir das considerações de Cavalcante e Costa (2011), para quem a institucionalização precoce e prolongada constitui-se em solo fértil para manifestação de estados de apatia e isolamento social.

No grupo Empatia, a questão referente a entrar em sintonia com o estado de ânimo de quem sofre obteve mais referências de menor pró-socialidade. Apesar dos comportamentos pró-sociais se tornarem relativamente estáveis no final da infância, durante a adolescência média (14-16 anos), os indivíduos teriam declínio em relação a sua percepção empática e conduta pró-social, havendo restabelecimento desses comportamentos em idades tardias (Auné et al., 2014; Gaspar, 2014). A empatia é tida como o principal motivador do comportamento pró-social, devido a suas características de compreensão do outro e preocupação com o próximo, e adolescentes menos empáticos tenderiam a maior instabilidade emocional e problemas de comportamento (Escrivá, García, \& Navarro, 2002; Mestre et al., 2007).

Os estudos sobre aspectos da adaptação social sobremaneira se voltam a investigações sobre os comportamentos antissociais e, também, sobre habilidades sociais. A investigação aqui exposta procurou trazer uma avaliação ainda pouco expressiva na literatura: a de comportamentos pró-sociais de adolescentes que têm contextos de desenvolvimento peculiares, o de instituições de acolhimento. Mesmo com limitações relativas ao tamanho da amostra, condição que não permite generalizações do estudo, os resultados encontrados na investigação foram, de certa forma, alinhados com tendências da literatura nacional e internacional na área da pró-socialidade.

Partindo da consideração da importância da adolescência para a constituição do desenvolvimento positivo nas demais etapas da vida, o trabalho ressaltou algumas condições a serem consideradas acerca do desenvolvimento de adolescentes que vivem em situação de acolhimento. Nesse sentido, o estudo apontou para elementos positivos da população estudada, considerada com histórico de vulnerabilidade, trazendo indicadores de potencialidades e de comportamentos positivos, no que tange à pró-socialidade.

Todavia se fazem necessárias mais investigações sobre o desenvolvimento dos adolescentes que vivem em serviços de acolhimento, de forma a ampliar e aprofundar avaliações que possam subsidiar intervenções voltadas à proteção dessa população, ainda para incrementar comportamentos pró-sociais. As condutas pró-sociais são importantes recursos para o enfrentamento das adversidades colocadas nos processos e contextos ambientais de desenvolvimento, em especial, a partir da saída desses adolescentes das instituições de acolhimento, o que se faz, obrigatoriamente, ao completarem 18 anos de idade.

\section{Referências}

Assis, S. G., \& Farias, L. O. P. (Eds.) (2013). Levantamento nacional das crianças e adolescentes em serviços de acolhimento. São Paulo: Hucitec.

Auné, S. E., Blum, D., Abal, F. J. P., Lozzia, G. S., \& Attorresi, H. F. (2014). La conducta prosocial: Estado actual de la investigación. Perspectivas en Psicología, 11(2), 21-33.

Aznar-Farias, M., \& Oliveira-Monteiro, N. R. O. (2006). Reflexões sobre pró-socialidade, resiliência e psicologia positiva. Revista Brasileira de Terapia Cognitiva, 2(2), 39-46.

Cáceda, R., Moskovciak, T., Prendes-Alvarez, S., Wojas, J., Engel, A., Wilker, S. H., Gamboa, J. L., \& Stowe, Z. N. (2014). Gender-specific effects of depression and suicidal ideation in prosocial behaviors. PLoS ONE, 9(9), e108733. doi:10.1371/ journal.pone.0108733

Carlos, D. M., Ferriani, M. G. C., Silva, M. A. I., Roque, E. M. S. T., \& Vendruscolo, T. S. (2013). O acolhimento institucional como proteção a adolescentes vítimas de violência doméstica: Teoria ou prática? Revista Latino-Americana de Enfermagem, 21(2), 7 telas. doi: 10.1590/S0104-11692013000200015

Caprara, G. V., Steca, P., Zelli, A., \& Capanna, C. (2005). A new scale for measuring adults' prosocialness. European Journal of Psychological Assessment, 21(2), 77-89. doi: 10.1027/10155759.21.2.77

Cavalcante, L. I. C., \& Costa, L. N. (2011). Comportamento de cuidado entre crianças institucionalizadas: Aspectos conceituais e estudos empíricos. In C. M. C Magalhães, L. I. C. Cavalcante, F. A. R. Pontes, S. S. C. Silva, \& L. S. Corrêa (Orgs.), Contextos ecológicos do desenvolvimento humano I. Belém: Paka-Tatu.

Cavalcante, L. I. C., Costa, L. N., \& Magalhães, C. M. C. (2012). Caretaking behavior among siblings in children's shelters. Psicologia Reflexão e Critica, 25(1), 165-173. doi: 10.1590/ S0102-79722012000100020

Chapman, M., Wall, A., \& Barth, R. (2004). Children's voice: The perceptions of children in foster care. American Journal of Orthopsychiatry, 74(3), 293-304. doi: 10.1037/00029432.74.3.293

Dell'Aglio, D. D., Koller, S. H., \& Yunes, M. A. M. (2006). Resiliência e Psicologia Positiva: Interfaces do risco à proteção. São Paulo: Casa do Psicólogo. 
Dell'Aglio, D. D., \& Siqueira, A. C. (2010). Preditores de satisfação de vida de jovens em situação de vulnerabilidade no sul do Brasil. Revista Psicodebate: Psicologia, Cultura y Sociedade, 10, 213-230. Recuperado de https://dspace.palermo.edu/ojs/ index.php/psicodebate/article/view/397/180

Decreto Lei no 8.069/1990 (1990). Dispõe sobre o Estatuto da Criança e do Adolescente e dá outras providências. Diário Oficial da União, seção 1.

Decreto Lei no 12.010/2009 (2009). Dispõe sobre adoção; altera as Leis n 8.069, de 13 de julho de 1990 - Estatuto da Criança e do Adolescente, 8.560, de 29 de dezembro de 1992; revoga dispositivos da Lei $n^{\circ} 10.406$, de 10 de janeiro de 2002 - Código Civil, e da Consolidação das Leis do Trabalho - CLT, aprovada pelo Decreto-Lei $n^{\circ} 5.452$, de $1^{\circ}$ de maio de 1943; e dá outras providências. Diário Oficial da União, seção 1.

Fernandes, A. O., \& Oliveira-Monteiro, N. R. (2016). Psychological indicators and perceptions of adolescents in residential care. Paidéia, 26(63), 81-89. doi: 10.1590/1982-43272663201610

Eisenberg, N., Eggum, N. D., \& Di Giunta, L. D. (2010). Empathyrelated responding: Associations with prosocial behavior, aggression, and intergroup relations. Social Issues and Policy Review, 4(1), 143-180. doi: 10.1111/j.1751-2409.2010.01020.x

Eisenberg, N., \& Musen, P.H. (1989). The roots of prosocial behavior in children. Cambridge: Cambridge University.

Escrivá, M. V. M., Garcia, P. S., \& Navarro, M. D. F. (2002). Procesos cognitivos y emocionales predictores de la conducta prosocial y agresiva: La empatía como factor modulador. Psicothema, 14(2), 227-232. Recuperado de http:/www. psicothema.com/pdf/713.pdf

Fernandes, A. O., Oliveira-Monteiro, N. R., Spadari-Bratfisch, R. C., Nascimento, J. O. G., \& Montesano, F. T. (2015). Estresse em adolescentes abrigados. Adolescência \& Saúde, 12(4), 65-75. Recuperado de http://www.adolescenciaesaude.com/ detalhe_artigo.asp?id=536\#

Gaspar, A. D. (2014). Neurobiologia e psicologia da empatia: Pontos de partida para a investigação e intervenção da promoção da empatia. Povos e Culturas, 01, 27-42.

González, J. J. Z., Cuéllar, A. I., Miguel, J. M. T., \& Desfilis, E. S. (2009). El desarrollo de la identidad en la adolescencia y adultez emergente: Una comparación de la identidad global frente a la identidad en dominios específicos. Anales de Psicología, 25(2), 316-329. Recuperado de http://www.redalyc. org/pdf/167/16712958014.pdf

Koller, S. H., \& Bernardes, N. M. G. (1997). Desenvolvimento moral pró-social: Semelhanças e diferenças entre os modelos teóricos de Eisenberg e Kohlberg. Estudos de Psicologia, 2(2), 223-262. doi: 10.1590/S1413-294X1997000200002

Mestre, M. V., Tur, A. M., Samper, P., Nácher, M. J., \& Cortés, M. T. (2007). Estilos de crianza en la adolescencia y su relación con el comportamiento prosocial. Revista Latinoamericana de Psicologia, 39(2), 211-225. Recuperado de http://www.redalyc. org/articulo.oa? $\mathrm{id}=80539201$
Ministério do Desenvolvimento Social e Combate à Fome. (2006). Plano Nacional de Promoção, Proteção e Defesa do Direito de Crianças e Adolescentes à Convivência Familiar e Comunitária. Brasília: CONANDA/CNAS. Recuperado de http://www.sdh.gov.br/assuntos/criancas-e-adolescentes/ programas/pdf/plano-nacional-de-convivencia-familiar-e.pdf

Organización Mundial de la Salud. (1999). Programación para la salud y el desarrollo de los adolescentes: Informe de un Grupo d'Estudio OMS/FNUAP/UNICEF [Programming for adolescent health and development: Report of a WHO/UNFPA/ UNICEF study group]. (OMS: TRS/886). Ginebra: OMS.

Poletto, M., Koller, S. H., \& Dell'Aglio, D. D. (2009). Eventos estressores em crianças e adolescentes em situação de vulnerabilidade social de Porto Alegre. Ciência \& Saúde coletiva, 14(2), 455-466. doi: 10.1590/S141381232009000200014

Reppold, C. T., Gurgel, L. G., \& Hutz, C. S. (2016). Evidências de validade da Escala de Conduta Social para Adolescentes. Avaliação Psicológica, 15(1), 83-91. Recuperado de http:// pepsic.bvsalud.org/pdf/avp/v15n1/v15n1a10.pdf

Roche, O. R. (2007). Desarrollo de la inteligencia emocional y social desde los valores y actitudes prosociales em la escuela. Buenos Aires: Ciudad Nueva.

Roche, O. R. (2010). Prosocialidad nuevos desafios: Métodos y pautas para la optimización creativa del entorno. Buenos Aires: Ciudad Nueva.

Roche, O. R., \& Selva, C. (2010). Young prosocial animation. In O. R. Roche (Org.), Prosocialidad nuevos desafios: Métodos y pautas para la optimización creativa del entorno. Buenos Aires: Ciudad Nueva.

Santos, M. F., \& Bastos, A. C. S. (2002). Padrões de interação entre adolescentes e educadores num espaço institucional: Ressignificando trajetórias de risco. Psicologia: Reflexão $e$ Critica, 15(1), 45-52. doi: 10.1590/S0102-79722002000100006

Sapienza, G. (2006). Comportamento pró-social, práticas educativas parentais e rendimento acadêmico do adolescente (Dissertação de mestrado). Universidade Federal de São Paulo.

Save the Children. (2009). Keeping children out of harmful institutions: Why we should be investing in family-based care. London: Save the Children.

Senna, S. R. C. M., \& Dessen, M. A. (2012). Contribuições das teorias do desenvolvimento humano para a concepção contemporânea da adolescência. Psicologia: Teoria e Pesquisa, 28(1), 101-108. Recuperado de http:/www.scielo.br/pdf/ptp/ v28n1/13.pdf

Siqueira, A. C., \& Dell'Aglio, D. D. (2007). Retornando à família de origem: Fatores de risco e proteção no processo de reinserção familiar de uma adolescente institucionalizada. Revista Brasileira de Crescimento e Desenvolvimento Humano, 17(3), 134-146. Recuperado de http://pepsic.bvsalud.org/pdf/rbcdh/ v17n3/13.pdf 
Siqueira, A. C., Zoltowski, A. P., Giordani, J. P., Otero, T. M., \& Dell'Aglio, D. D. (2010). Processo de reinserção familiar: Estudo de casos de adolescentes que viveram em instituição de abrigo. Estudos de Psicologia, 15(1), 7-15. doi: 10.1590/ S1413-294X2010000100002

Steinberg, L., \& Lerner, R. M. (2004). The scientific study of adolescence: A brief history. The Journal of Early Adolescence, 24(1), 45-54. doi: 10.1177/0272431603260879
Vecchione, M. V., \& Piconni, L. (2010). Differenze di età e di genere nella condotta prosociale. In G. V. Caprara, \& S. Bonino (Orgs.), Il comportamento prosociale: Aspetti individuali, familiar e social. Trento: Erickson.

Wathier, J. L., \& Dell'Aglio, D. D. (2007). Sintomas depressivos e eventos estressores em crianças e adolescentes no contexto de institucionalização. Revista de Psiquiatria do Rio Grande do Sul, 29(3), 305-314. doi: 10.1590/S0101-81082007000300010

Yunes, M. A. M., Miranda, A. T., \& Cuello, S. E. S. (2004). Um olhar ecológico para os riscos e as oportunidades de desenvolvimento de crianças e adolescentes. In S. H. Koller (Org.), Ecologia do desenvolvimento humano: Pesquisa e intervenção no Brasil. São Paulo: Casa do Psicólogo.

Recebido em 10.04.2013

Primeira decisão editorial em 18.04.2016

Versão final em 17.05.2016

Aceito em 19.07.2016 